\title{
Endothelin in human cerebrovascular nerves
}

\author{
Andrzej LOESCH ${ }^{*}$ and Geoffrey BURNSTOCK $†$ \\ *Department of Anatomy and Developmental Biology and Centre for Neuroscience, University College London, Gower Street, \\ London WCIE 6BT, U.K., and †Autonomic Neuroscience Institute, Royal Free and University College Medical School, Rowland \\ Hill Street, London NW3 2PF, U.K.
}

\begin{abstract}
A
Novel data is presented from this electron-immunocytochemical study that demonstrates endothelin-containing cerebrovascular nerves in the human middle cerebral artery.
\end{abstract}

\section{INTRODUCTION}

It is commonly known that endothelin-1 (ET-1) has potent vasoconstrictor, hypertrophic, mitogenic and anti-apoptotic effects on vascular smooth muscle [1-3]. Using electron-immunocytochemical techniques, we have revealed the presence of ET-1 in a subpopulation of endothelial cells of large cerebral arteries, such as basilar and posterior communicating artery in rabbit and/or rat, as well as in human postmortem middle cerebral artery (MCA) [4-6]. Release studies have demonstrated that changing the rate of cerebrovascular perfusion with perfluorocarbon emulsion (a substitute for erythrocytes to deliver oxygen to the brain) causes changes in ET-1 content in the effluents from the rabbit brain [7]. Hence, it has been shown that abrupt increases in flow, producing shear stress, induced increased ET-1 levels in the effluents from brain suggesting an endothelial origin of ET-1 in the cerebral circulation [7]. Furthermore, increases in cerebral flow are accompanied by a decrease in endothelial immunoreactivity to ET-1 [4].

In addition to an endothelial source of ET-1 in large cerebral arteries, an ultrastructural study of immunoreactivity to this peptide has also revealed the presence of ET-1-positive perivascular nerves in the healthy rat basilar artery [8]. A similar study of the basilar artery of spontaneously hypertensive rats has demonstrated an increase in ET-1-positive perivascular axons, although these axons might be degenerating [9].

In the present electron-immunocytochemical study, we report for the first time that ET-1 is localized in cerebrovascular nerves of the human (post-mortem) MCA.

\section{MATERIALS AND METHODS}

Both ethical approval and patient informed consent were obtained for this study. A human autopsy specimen of the left M2 segment of the MCA was obtained from a female subject (age 59 years) with no apparent cerebrovascular and/or cerebral disease, at postmortem time 35 h. Post-mortem examination concluded that coronary occlusion was a cause of death. The MCA was initially placed in Hanks solution for about $1 \mathrm{~h}$, and then fixed overnight in $4 \%(\mathrm{v} / \mathrm{v})$ paraformaldehyde and $0.2 \%$ glutaraldehyde (in 0.1 M phosphate buffer, $\mathrm{pH}$ 7.4) at $4{ }^{\circ} \mathrm{C}$. The MCA was then washed in phosphate buffer and $0.1 \mathrm{M}$ Tris buffer ( $\mathrm{pH}$ 7.6). Approx 5-mm long strips of MCA (obtained following longitudinal section) were then processed for ET-1 peroxidase-antiperoxidase (PAP) immunoprocedure using the protocol reported previously [5]. In brief, specimens were (i) exposed to $0.3 \%$ hydrogen peroxide in $50 \%(\mathrm{v} / \mathrm{v})$ methanol for $30 \mathrm{~min}$, (ii) exposed for $1.5 \mathrm{~h}$ to $10 \%(\mathrm{v} / \mathrm{v})$ normal goat serum (Nordic Immunology, Tilberg, The Netherlands), (iii) incubated for $48 \mathrm{~h}$ at $4{ }^{\circ} \mathrm{C}$ with a rabbit polyclonal antibody to ET-1 at a dilution 1:1000, (iv) exposed to goat anti-rabbit IgG (Biogenesis, Bournemouth, Dorset, U.K.) diluted 1:40, (v) incubated for $3 \mathrm{~h}$ with a rabbit PAP complex (DAKO, Glostrup, Denmark) diluted 1:75, (vi) treated with 3,3'-diaminobenzidine (Sigma,

Key words: endothelin, cerebrovascular nerves, human.

Abbreviations: ET, endothelin; MCA, middle cerebral artery; PAP, peroxidase-antiperoxidase.

Correspondence: Dr A. Loesch (e-mail a.loesch@ucl.ac.uk). 


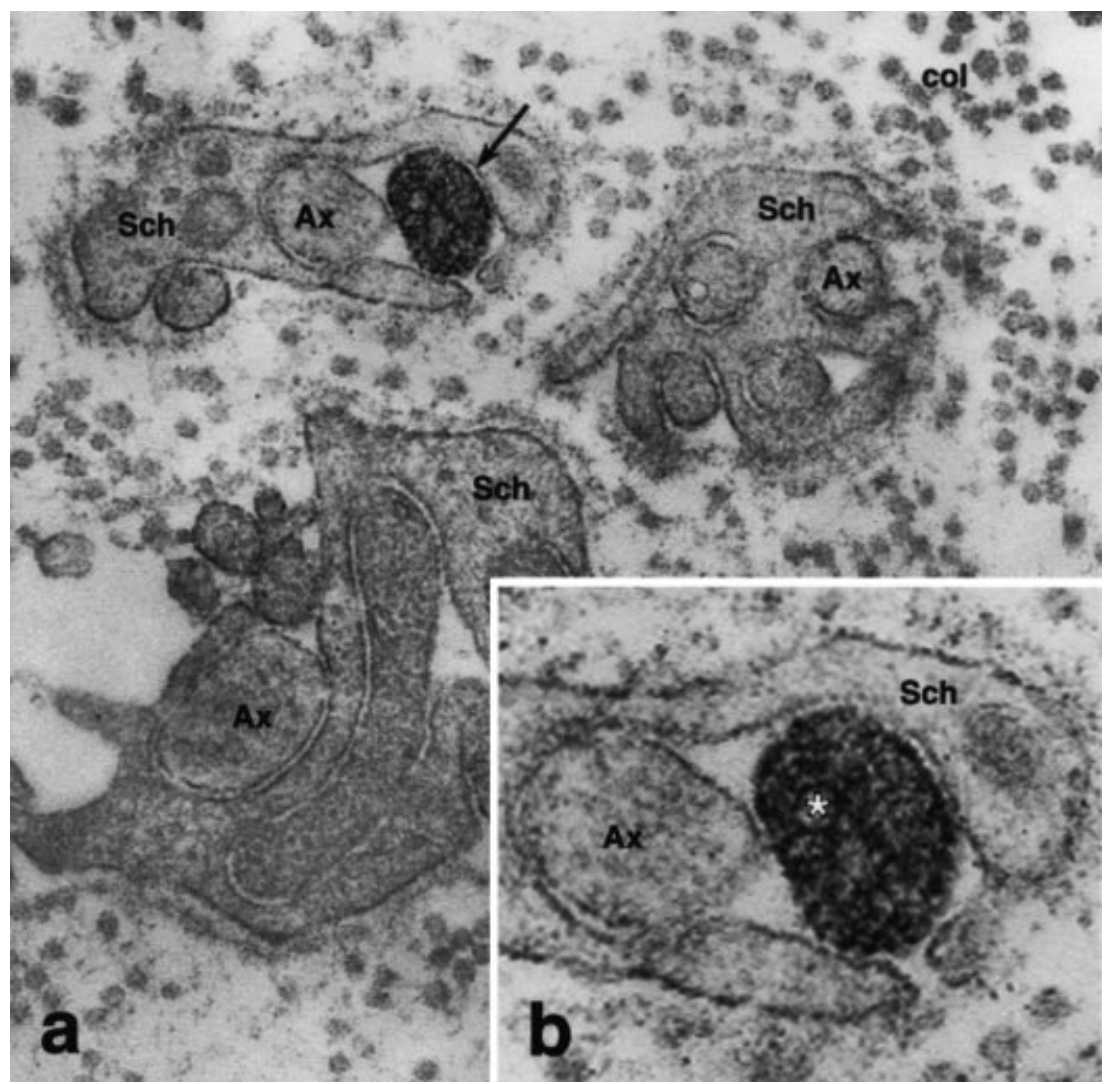

Figure I Electron-immunocytochemical staining of perivascular nerve fibres of the human middle cerebral artery labelled for ET-I

(a) One ET-I-positive (arrow) and a few ET-I-negative (Ax) axon profiles are seen in the outer plexus (some distance from the vascular smooth muscle of the media). Schwann cells (Sch) and collagen (col) can also be seen. Magnification: $42500 \times$. (b) A magnified fragment of the innervation illustrated in (a) shows that in the labelled axon, the immunoprecipitate is densely packed in the axoplasm and also in association with intra-axonal structures, such as the outer membrane of mitochondria (asterisk). Magnification: $69700 \times$.

Poole, Dorset, U.K.) and $0.01 \%$ hydrogen peroxide, (vii) post-fixed with $1 \%(\mathrm{w} / \mathrm{v})$ osmium tetroxide (for $1 \mathrm{~h}$ at $4{ }^{\circ} \mathrm{C}$ ), (viii) dehydrated in a graded series of ethanol and propylene oxide, and embedded in Araldite. Ultrathin circumferential sections were contrasted with uranyl acetate and lead citrate and subsequently examined in JEOL-1010 electron microscope.

\section{Controls}

Rabbit polyclonal ET-1 antibody to human/porcine ET-1 was manufactured and characterized by Cambridge Research Biochemicals (CRB, Cambridge, U.K.). It has previously been used in PAP electron-immunocytochemical studies of animal and human endothelial cells $[5,10]$, as well as in immunoassay to detect ET-1 from cultured endothelial cells [11]. CRB reported preincubation of ET-1 antibody with 10 nmol human ET-1, ET-2 or ET-3 substances per $\mathrm{ml}$ of optimal diluted antibody was sufficient to abolish immunolabelling. Our own studies showed that preabsorption of ET-1 antibody with its respective antigen (synthetic human ET-1, CRB) eliminated positive labelling in endothelial cells [10]. Immunoassay of ET-1 antibody showed that this antibody cross-reacted with big endothelin 39 (7\%), ET-2 $(15 \%)$ and ET-3 (100\%) [12]. In the present study, the specificity of the immunolabelling was investigated routinely by omission of the ET-1 antibody and IgG steps, independently, as well as by replacement of ET-1 antibody by non-immune normal rabbit serum (Nordic Immunology). No labelling was observed in these controls.

\section{RESULTS}

Immunoreactivity for ET-1 ('black' immunoprecipitate seen at the electron microscope level) was observed in a subpopulation of axon profiles of perivascular nerves of human post-mortem MCA (Figures 1a and 1b). These axons were located in nerve bundles in the outer 
adventitia, some distance from the smooth muscle of the media. Immunoprecipitate in labelled axons was associated with the axoplasm and membrane of intracellular structures, such as mitochondria (Figure 1b). The ET-1positive axons were seen in association with unlabelled axon profiles and Schwann cell processes. The ET-1 antibody used in the present study also immunoreacted with endothelial cells of MCA examined (results not shown). No labelling was seen in controls (see the Materials and methods section).

\section{DISCUSSION}

The present case report shows new data regarding human cerebral blood vessels. We demonstrated that perivascular nerves of human MCA immunoreact with an antibody raised to recognized human ET-1. The present study, therefore, is consistent with our earlier observations of immunoreactive ET-1 in cerebrovascular nerves of rat basilar artery $[8,9]$.

In the present study, no data is available on the type of synaptic vesicles that are present in varicosities of ET-1positive cerebrovascular nerves. No immunolabelled varicosities located close to the vascular smooth muscle of the media were observed. This, however, does not exclude the possibility that ET-1-positive varicosities can be located in the inner nerve plexus, near vascular smooth muscle. Limitation of the labelling could be caused by, for example, poor infiltration of immunoreagents to the adventitial-medial border. Our previous studies, carried out on rat basilar artery (which contains less adventitial connective tissue than human MCA), revealed that ET-1positive varicosities usually contain small spherical agranular vesicles $(42 \mathrm{~nm})$ and a few large granular vesicles $(92 \mathrm{~nm})$ [8]. As a matter of interest, our previous study of human MCA detected type-1 nitric oxide synthase-immunoreactive varicosities in the adventitia, which dominated in nerve profiles containing small agranular synaptic vesicles [5].

As yet, the role that ET-1 could play in cerebrovascular nerves is unknown. A recent study by Milner and colleagues [9] suggests that ET from cerebrovascular nerves may be implicated in the development of hypertension at least in cerebral arteries of spontaneously hypertensive rats, since an increased presence of ET-1positive nerves to these arteries was observed. ET-positive varicosities of cerebrovascular nerves in hypertensive animals displayed structural abnormalities which suggested that these nerves may be undergoing degenerative changes [9]. A role of ET-1 in repair of damaged neurones has therefore been suggested [13].

A study of rats, following sensory and sympathetic denervation, suggested that the ET-positive perivascular nerves of large cerebral arteries may be the projections of primary afferent sensory neurones of trigeminal ganglia expressing ET-1 mRNA [13]. This raises possibility that ET-1 may co-act with other neuropeptides, such as substance $\mathrm{P}$ and calcitonin gene-related peptide, known to be present in trigeminal ganglia sensory nerves innervating large cerebral vessels [14]. Further studies are required to establish if ET-1 in cerebrovascular nerves acts as a transmitter, co-transmitter or neuromodulator and what effects it produces on cerebral arteries. Pharmacological evidence for the action of ET-1 as a neuromodulator in sensory neurones has been known for some time [15], while studies on the rat heart supported a role for ET-1 as a sensory-motor neurotransmitter [16]. On the other hand, there is evidence to suggest that ET-1 can also be synthesized and released from postganglionic sympathetic neurones, including those of the rat superior cervical ganglion that projects to cerebral vessels $[9,13,17]$.

Since the ET-1 antibody (raised against human ET-1) used in the present study may also recognize ET-2 and ET-3 to lesser extent [12], the possibility cannot be excluded that we are observing labelling not only for ET-1 but also for ET-2 and ET-3. It should be pointed out, however, that the synthesis of ET-1 in the neurones of trigeminal ganglia projecting to cerebral arteries was confirmed by the detection of ET-1 mRNA [9]. In the present study, detection of ET-1-positive endothelial cells in the intima of MCA (results not shown) indicates specificity of the immunolabelling for ET-1, even if the antibody is also able to recognize big ET, ET-2 or ET-3.

In conclusion, ET-1 of neural-perivascular origin may play a physiological role in cerebral arteries, as may the ET-1 originating from cerebrovascular endothelial cells. It should be mentioned that cerebral arteries receive rich innervation comprising sensory, sympathetic and parasympathetic neurotransmitters/neuromodulators, including type-1 nitric oxide synthase-containing vasodilator nerves that produce nitric oxide $[18,19]$. A variety of vasoactive agents, as well as NOS, have also been identified in cerebrovascular endothelium making cerebral vessels well equipped with sensitive mechanisms to control local blood flow $[18,20]$. It will be important to establish the role of ET-1 of neural origin in these mechanisms. As the current report on ET-1 in human MCA is a case report, it is obvious that further ultrastructural and immunocytochemical studies of ET-1-containing cerebrovascular nerves in human cerebral vessels of different sizes in different regions of the brain are desirable. Such studies are currently in progress in our laboratories. Immunocytochemistry of vibratome sections of cerebral vessels is expected to provide more data about the distribution of ET-1-containing cerebrovascular nerves and their association with vasoactive agents that code sensory, sympathetic or parasympathetic nerves, as well as changes in the distribution of these nerves in different disease conditions. 


\section{REFERENCES}

1 Tasaka, K. and Kitazumi, K. (1994) The control of endothelin-1 secretion. Gen. Pharmacol. 25, 1056-1069

2 Sharifi, A. M. and Schiffrin, E. L. (1997) Apoptosis in aorta of deoxycorticosterone acetate-salt hypertensive rats: effect of endothelin receptor antagonism. J. Hypertens. 15, 1441-1448

3 Komuro, I., Kurihara, H., Sugiyama, T., Yoshizumi, M., Takaku, F. and Yazaki, Y. (1988) Endothelin stimulates $\mathrm{c}$-fos and c-myc expression and proliferation of vascular smooth muscle cells. FEBS Lett. 238, 249-252

4 Loesch, A., Domer, F. R., Alexander, B. and Burnstock, G. (1993) Electron-immunocytochemistry of peptides in endothelial cells of rabbit cerebral vessels following perfusion with a perfluorocarbon emulsion. Brain Res. 611, 333-337

5 Gorelova, E., Loesch, A., Bodin, L., Chadwick, L. and Burnstock, G. (1996) Localization of immunoreactive Factor VIII, nitric oxide synthase, substance P, endothelin-1 and 5-hydroxytryptamine in human postmortem middle cerebral artery. J. Anat. 188, 97-107

6 Shochina, M., Loesch, A., Rubino, A., Miah, S., Macdonald, G. and Burnstock, G. (1997)

Immunoreactivity for nitric oxide synthase and endothelin in the coronary and basilar arteries of renal hypertensive rats. Cell Tiss. Res. 288, 509-516

7 Domer, F. R., Alexander, B., Milner, P., Bodin, P. and Burnstock, G. (1993) Effect of changes in rate of vascular perfusion on release of substances into the effluent from the brain of the rabbit. Brain Res. 630, 88-94

8 Loesch, A., Milner, P. and Burnstock, G. (1998) Endothelin in perivascular nerves. An electronimmunocytochemical study of rat basilar artery. Neuroreport 9, 3903-3906

9 Milner, P., Loesch, A. and Burnstock, G. (2000) Neural endothelin in hypertension: increased immunoreactivity in ganglia and nerves to cerebral arteries of the spontaneously hypertensive rat. J. Vasc. Res. 37, 39-49
10 Loesch, A., Bodin, B. and Burnstock, G. (1991)

Colocalization of endothelin, vasopressin and serotonin in cultured endothelial cells of rabbit aorta. Peptides 12, 1095-1103

11 Milner, P., Bodin, P., Loesch, A. and Burnstock, G. (1990) Rapid release of ATP from isolated aortic endothelial cells exposed to increased flow. Biochem. Biophys. Res. Commun. 170, 649-656

12 Bodin, P., Milner, P., Winter, R. and Burnstock, G. (1992) Chronic hypoxia changes the ratio of endothelin to ATP release from rat aortic endothelial cells exposed to high flow. Proc. Royal Soc. Lond. B Biol. Sci. 247, 131-135

13 Milner, P., Loesch, A. and Burnstock, G. (2000) Endothelin immunoreactivity and mRNA expression in sensory and sympathetic neurones following selective denervation. Int. J. Dev. Neurosci. 18, 722-734

14 Suzuki, N., Hardebo, J. E. and Owman, C. (1989) Origins and pathways of cerebrovascular nerves storing substance $\mathrm{P}$ and calcitonin gene-related peptide in rat. Neuroscience 31, 427-438

15 Dymshitz, J. and Vasko, M. R. (1991) Endothelin-1 enhances capsaicin-induced peptide release and cGMP accumulation in cultures of rat sensory neurones. Neurosci. Lett. 167, 128-132

16 Szolcsanyi, J., Oroszi, G., Nemeth, J., Szilvássy, Z. and Tósaki, A. (1999) Endothelin release by capsaicin in isolated working rat heart. Eur. J. Pharmacol. 376, 247-250

17 Damon, D. H. (1998) Postganglionic sympathetic neurons express endothelin. Am. J. Physiol. 274, R873-R878

18 Burnstock, G. (1990) Local mechanisms of blood flow control by perivascular nerves and endothelium. J. Hypertens. 8 (Suppl.), S95-S106

19 Burnstock, G. and Ralevic, V. (1994) New insights into the local regulation of blood flow by perivascular nerves and endothelium. Br. J. Plastic Surg. 47, 525-543

20 Loesch, A. and Burnstock, G. (1996) Immunocytochemistry of vasoactive agents and nitric oxide synthase in vascular endothelial cells with emphasis on the cerebral blood vessels. Cell Vision 3, 346-357 